\title{
GENERATORS FOR SOME RINGS OF ANALYTIC FUNCTIONS
}

\author{
BY LARS HÖRMANDER
}

Communicated by R. C. Buck, July 10, 1967

Let $\Omega$ be an open set in $C^{n}$ and let $p$ be a nonnegative function defined in $\Omega$. We shall denote by $A_{p}(\Omega)$ the set of all analytic functions $f$ in $\Omega$ such that for some constants $C_{1}$ and $C_{2}$

$$
|f(z)| \leqq C_{1} \exp \left(C_{2} p(z)\right), \quad z \in \Omega .
$$

It is obvious that $A_{p}(\Omega)$ is a ring. We wish to determine when it is generated by a given finite set of elements $f_{1}, \cdots, f_{N}$. There is an obvious necessary condition, for if $f_{1}, \ldots, f_{N}$ are generators for $A_{p}(\Omega)$ we can in particular find $g_{1}, \cdots, g_{N} \in A_{p}(\Omega)$ so that $1=\sum f_{j} g_{j}$. Hence we have

$$
1 \leqq \sum\left|f_{j}(z)\right| C_{1} \exp \left(C_{2} p(z)\right)
$$

for some constants $C_{1}$ and $C_{2}$, that is,

$$
\left|f_{1}(z)\right|+\cdots+\left|f_{N}(z)\right| \geqq c_{1} \exp \left(-c_{2} p(z)\right), \quad z \in \Omega,
$$

for some positive constants $c_{1}$ and $c_{2}$.

This note concerns the converse statement. Carleson [1] has proved a deep result of that type, called the Corona Theorem, which states that (2) implies that $f_{1}, \cdots, f_{N}$ generate $A_{p}(\Omega)$ if $p=0$ and $\Omega$ is the unit disc in $C$. In a recent research announcement [5] in this Bulletin, the Corona Theorem was used to prove the analogous result when $p(z)=|z|$ and $\Omega=C$. However, we shall see here that this statement is much more elementary than the Corona Theorem; indeed, we shall prove a general result of this kind for functions of several complex variables although no analogue of the Corona Theorem is known there.

THEOREM 1. Let $p$ be a plurisubharmonic function in the open set $\Omega \subset C^{n}$ such that

(i) all polynomials belong to $A_{p}(\Omega)$;

(ii) there exist constants $K_{1}, \cdots, K_{4}$ such that $z \in \Omega$ and $|z-\zeta|$ $\leqq \exp \left(-K_{1} p(z)-K_{2}\right) \Rightarrow \zeta \in \Omega$ and $p(\zeta) \leqq K_{3} p(z)+K_{4}$.

Then $f_{1}, \cdots, f_{N} \in A_{p}(\Omega)$ generate $A_{p}(\Omega)$ if and only if $(2)$ is valid.

Before the proof we make a few remarks. First note that if $d(z)$ 
denotes the distance from $z \in \Omega$ to $C \Omega$ then (ii) implies that $d(z)$ $\geqq \exp \left(-K_{1} p(z)-K_{2}\right)$, that is,

$$
p(z) \geqq\left(\log 1 / d(z)-K_{2}\right) / K_{1} .
$$

Hence $p(z) \rightarrow \infty$ if $z$ converges to a boundary point of $\Omega$, so $\Omega$ is pseudoconvex and therefore a domain of holomorphy (cf. [3, Theorem 4.2.8]). On the other hand, if $\Omega$ is a domain of holomorphy it follows that $p(z)=\log 1 / d(z)$ is plurisubharmonic, and (ii) is valid with $K_{1}=K_{3}=1$ and suitable $K_{2}, K_{4}$. Another example is obtained by taking $p(z)=\sum\left|z_{j}\right|^{p}, \Omega=C^{n}$, where $p$ is any positive number. When $n=1$ this yields the results announced in [5]. However, the Corona Theorem is not contained in Theorem 1 but will be discussed at the end of the note.

We know already that (2) is a necessary condition for $f_{1}, \cdots, f_{N}$ to be generators. To prove the sufficiency we shall apply a standard homological argument (cf. e.g. Malgrange [6]) but first a few lemmas are required.

LeMma 2. If $f \in A_{p}(\Omega)$ it follows that $\partial f / \partial z_{j} \in A_{p}(\Omega)$.

Proof. From (1) and (ii) we obtain

$|f(\zeta)| \leqq C_{1} \exp \left(C_{2}\left(K_{3} p(\xi)+K_{4}\right)\right)$ if $|\zeta-z| \leqq \exp \left(-K_{1} p(z)-K_{2}\right)$.

Hence

$$
\left|\partial f(z) / \partial z_{j}\right| \leqq C_{1} \exp \left(C_{2}\left(K_{3} p(z)+K_{4}\right)+K_{1} p(z)+K_{2}\right) .
$$

Since we shall use $\bar{\partial}$ cohomology with bounds in $L^{2}$ norms, we also note that the definition of $A_{p}(\Omega)$ can be expressed in terms of such norms.

Lemma 3. If $f$ is analytic in $\Omega$, then $f \in A_{p}(\Omega)$ if and only if for some $K$

$$
\int|f|^{2} e^{-2 K_{p}} d \lambda<\infty
$$

where $d \lambda$ denotes the Lebesgue measure.

Proof. If (1) is valid we obtain (3) since $(1+|z|)^{2 n+1} \leqq B_{1} \exp B_{2} p(z)$ in view of (i). On the other hand, it follows from (3) and (ii) that the mean value of $|f|$ over the ball $\left\{\zeta ;|\zeta-z| \leqq \exp \left(-K_{1} p(z)-K_{2}\right)\right\}$ is bounded by $C \exp \left(K\left(K_{3} p(z)+K_{4}\right)+2 n\left(K_{1} p(z)+K_{2}\right)\right)$. Since this is also a bound for $|f(z)|$, the lemma is proved. 
Lemma 4. Let $g$ be a form of type $(0, r+1)$ in $\Omega$ with locally square integrable coefficients and $\bar{\partial} g=0$, and let $\phi$ be a plurisubharmonic function in $\Omega$ such that

$$
\int|g|^{2} e^{-\phi} d \lambda<\infty .
$$

If $r \geqq 0$ it follows that there is a form $f$ of type $(0, r)$ with $\bar{\partial} f=g$ and

$$
\int|f|^{2} e^{-\phi}\left(1+|z|^{2}\right)^{-2} d \lambda \leqq \int|g|^{2} e^{-\phi} d \lambda .
$$

The norms here are defined as in $\$ 4.1$ of [3]. The lemma follows from Theorem $2.2 .1^{\prime}$ in [2] by the argument used in [3] to derive Theorem 4.4.2 from Theorem 4.4.1.

For nonnegative integers $s$ and $r$ we shall denote by $L_{r}^{s}$ the set of all differential forms $h$ of type $(0, r)$ with values in $\Lambda^{8} C^{N}$, such that for some $K$

$$
\int|h|^{2} e^{-2 K_{p}} d \lambda<\infty .
$$

In other words, for each multi-index $I=\left(i_{1}, \cdots, i_{8}\right)$ of length $|I|=s$ with indices between 1 and $N$ inclusively, $h$ has a component $h_{I}$ which is a differential form of type $(0, r)$ such that $h_{I}$ is skew symmetric in $I$ and

$$
\int\left|h_{I}\right|^{2} e^{-2 K p} d \lambda<\infty
$$

The $\bar{\partial}$ operator defines an unbounded map from $L_{r}^{s}$ to $L_{r+1}^{s}$; its domain consists of all $h \in L_{r}^{s}$ such that $\bar{\partial} h$, defined in the sense of distribution theory with $\bar{\partial}$ acting on each component $h_{I}$ is an element of $L_{r+1}^{s}$. Furthermore, the interior product $P_{f}$ by $\left(f_{1}, \cdots, f_{N}\right)$ maps $L_{r}^{s+1}$ into $L_{r}^{s}:$ If $h \in L_{r}^{s+1}$ then

$$
\left(P_{f} h\right)_{I}=\sum_{1}^{N} h_{I j} f_{j}, \quad|I|=s .
$$

We define $P_{f} L_{r}^{0}=0$. Clearly $P_{f}^{2}=0$ and $P_{f}$ commutes with $\bar{\partial}$ since $f_{j}$ are analytic, so we have a double complex.

LeMma 5. The equation $\bar{\partial} g=h$ has a solution $g \in L_{r}^{s}$ for every $h \in L_{r+1}^{s}$ with $\bar{\partial} h=0$.

Proof. In view of (i) this is an immediate consequence of Lemma 4. 
Lemma 6. If $g \in L_{r}^{s}$ and $P_{f} g=0$, we can find $h \in L_{r}^{s+1}$ such that $g=P_{f} h$ and in addition $\bar{\partial} h \in L_{r+1}^{s+1}$ if $\bar{\partial} g=0$.

Proof. We can take for $h$ essentially the exterior product of $g$ by $\bar{f} /|f|^{2}$. More precisely, we set when $|I|=s+1$

$$
h_{I}=\sum_{1}^{s+1} g_{I_{j}}(-1)^{s 1-j \bar{f}^{+}} i_{j} /|f|^{2},
$$

where $I_{j}$ denotes the multi-index $I=\left(i_{1}, \cdots, i_{s+1}\right)$ with the index $i_{j}$ removed. It follows from (2) that $h \in L_{r}^{s+1}$, and since $P_{f} g=0$ it is obvious that $P_{f} h=g$. If $\bar{\partial} g=0$ we can compute $\bar{\partial} h_{I}$ by operating on the factor $\bar{f}_{i,} /|f|^{2}$ alone, so it follows from (2) and Lemma 2 that $\bar{\partial} h \in L_{r+1}^{s+1}$.

It is now easy to prove the following theorem which in view of Lemma 3 contains Theorem 1 for $r=s=0$. (Actually Theorems 1 and 7 are equivalent.)

THEOREM 7. For every $g \in L_{r}$ with $\bar{\partial} g=P_{f} g=0$ one can find $h \in L_{r}^{s+1}$ so that $\bar{\partial} h=0$ and $P_{f} h=g$.

Proof. The theorem is trivially valid when $r>n$ or $s>N$. In the proof we may therefore assume that it has already been established for larger values of $r$ and $s$. By Lemma 6 we can find $h^{\prime} \in L_{r}^{s+1}$ so that

$$
P_{f} h^{\prime}=g, \quad \bar{\partial} h^{\prime} \in L_{r+1}^{s+1}
$$

Since $\bar{\partial} \bar{\partial} h^{\prime}=0$ and $P_{f} \bar{\partial} h^{\prime}=\bar{\partial} P_{f} h^{\prime}=\bar{\partial} g=0$, it follows from the inductive hypothesis that one can find $h^{\prime \prime} \in L_{r+1}^{s+2}$ such that

$$
P_{f} h^{\prime \prime}=\bar{\partial} h^{\prime}, \quad \bar{\partial} h^{\prime \prime}=0 .
$$

By Lemma 5 we can find $h^{\prime \prime \prime} \in L_{r}^{s+2}$ so that $\bar{\partial} h^{\prime \prime \prime}=h^{\prime \prime}$. If $h=h^{\prime}-P_{f} h^{\prime \prime \prime}$ we conclude that $\bar{\partial} h=\bar{\partial} h^{\prime}-P_{f} \bar{\partial} h^{\prime \prime \prime}=\bar{\partial} h^{\prime}-P_{f} h^{\prime \prime}=0$, and that $P_{f} h$ $=P_{f} h^{\prime}=g$. The proof is complete.

We shall end this note by showing how the proofs of Carleson [1] can be adapted to the conventional pattern used in the proof of Theorem 1. This does not remove the main difficulties but it does eliminate a tricky argument due to D. J. Newman, which was used in [1] in the case of more than 2 generators. In the proof of Theorem 1 the main points were the existence theorems for the operators $\bar{\partial}$ and $P_{f}$ given in Lemmas 5 and 6 . The proof of the Corona Theorem requires a more precise version of both.

From now on $\Omega$ will denote the unit disc in $C$. (All the arguments are valid for any bounded open set in $C$ with a $C^{2}$ boundary.) If $\mu$ is a 
bounded measure in $\Omega$ and $\phi$ is an integrable function on $\partial \Omega$, we shall say that a distribution in $\Omega$ satisfying the Cauchy-Riemann equation

$$
\partial u / \partial \bar{z}=\mu \text { in } \Omega
$$

has boundary values $\phi$ on $\partial \Omega$ provided that there exists a distribution $U$ with support in $\bar{\Omega}$ such that $U=u$ in $\Omega$ and

$$
\partial U / \partial \bar{z}=\mu-\phi d z / 2 i \text {. }
$$

Here $\phi d z$ is of course a measure on $\partial \Omega$, and $\mu$ is extended so that there is no mass in the complement of $\Omega$. If $u=0$ it follows from (6) that $U=0$, for $\partial U / \partial \bar{z}$ would otherwise be a distribution with support on $\partial \Omega$ with positive transversal order. Hence $u$ determines both $\mu, \phi$ and $U$ uniquely, so it is legitimate for us to say that $\phi$ is the boundary value of $u$.

If $u$ belongs to the Hardy class $H^{p}$ for some $p \geqq 1$, then $\phi$ coincides a.e. with the boundary values in the usual sense, and $\mu=0$. Conversely, if $u$ is analytic and has boundary values belonging to $L^{p}(\partial \Omega)$ in the sense of (6), it follows that $u \in H^{p}(p \geqq 1)$. If $f \in H^{\infty}$ and $u$ is a solution of (5) with boundary values $\phi$, then $f u$ satisfies (5) with $\mu$ replaced by $f \mu$ and has boundary values $f \phi$. This is obvious when $f$ is analytic in a neighborhood of $\bar{\Omega}$ and follows in general if we first consider $f(r z)$ with $r<1$ and then let $r \rightarrow 1$, noting that the solution $U \in \mathcal{E}^{\prime}(\bar{\Omega})$ of the equation $\partial U / \partial \bar{z}=F$ is a continuous function of $F \in \mathcal{E}^{\prime}(\bar{\Omega})$ when it exists.

The existence of a solution of (6) with support in $\bar{\Omega}$ means precisely that the right hand side is orthogonal to all (entire) analytic functions. Thus (5) has a solution with boundary values $\phi$ if and only if for entire analytic $f$

$$
\int f d \mu=(2 i)^{-1} \int \phi(z) f(z) d z .
$$

In view of the Hahn-Banach Theorem it follows that there exists a solution with boundary values of absolute value $\leqq C$ if and only if for entire analytic $f$

$$
\left|\int f d \mu\right| \leqq C \int|f(z)||d z| / 2 .
$$

A sufficient condition for this is given by the following result of [1]. (See also [4] where an extension to several variables is given.)

Lemma 8. There is a constant $C$ such that 
(7) $\int_{\Omega}|v(z)|^{p}|d \mu(z)| \leqq C M \int_{\partial \Omega}|v|^{p}|d z|, \quad v \in H^{p}(\Omega), p>0$,

for every measure $\mu$ in $\Omega$ such that

$$
|\mu|\{\zeta ;|\zeta-z|<r\} \leqq M r, \quad z \in \partial \Omega, r>0 .
$$

We now modify the definition of $L_{r}^{s}$ as follows:

$h \in L_{0}^{s}$ if $\partial h_{I} / \partial \bar{z}$ is a bounded measure in $\Omega$ and $h_{I}$ has boundary values in $L^{\infty}(\partial \Omega),|I|=s ; h \in L_{1}^{s}$ if $h_{I}=\mu_{I} d \bar{z}$ where $\mu_{I}$ is a measure in $\Omega$ satisfying (8), $|I|=s$. Of course we take $L_{r}^{s}=0$ when $r>1$. From Lemma 8 and the discussion preceding it we conclude that Lemma 5 remains valid and that $\left\{h ; h \in L_{0}^{0}, \bar{\partial} h=0\right\}=H^{\infty}$.

Let $f_{j} \in H^{\infty}, j=1, \cdots, N$, and assume that for some $c>0$

$$
\left|f_{1}(z)\right|+\cdots+\left|f_{N}(z)\right| \geqq c .
$$

If we define $P_{f}$ by means of these functions, the proof of Lemma 6 remains valid when $s=1$ but breaks down when $s=0$ since $\partial f_{j} / \partial z$ need not be a bounded function. We must therefore use another construction, based on the following

Lemma 9. For sufficiently small $\epsilon>0$ one can find a partition of unity $\phi_{j}$ subordinate to the covering of $\Omega$ by the open sets $\Omega_{j}=\left\{z ;\left|f_{j}(z)\right|>\epsilon\right\}$ such that $\partial \phi_{j} / \partial \bar{z}$, defined in the sense of distribution theory, is a measure which satisfies (8) for all $j$ and some $M$.

Admitting Lemma 9 for a moment we shall see that it implies the Corona Theorem. With our new definition of $L_{r}$ we have already seen that Lemma 5 remains valid as well as Lemma 6 for $r \neq 0$. To prove Lemma 6 for $r=0$ we need only replace $\bar{f}_{j} /|f|^{2}$ in the previous proof by $\phi_{j} / f_{j}$ where $\phi_{j}$ is the partition of unity in Lemma 9. In fact, $\partial\left(\phi_{j} / f_{j}\right) / \partial \bar{z}=f_{j}^{-1} \partial \phi_{j} / \partial \bar{z}$ satisfies (8) since $\left|f_{j}\right| \geqq \epsilon$ in supp $\phi_{j}$. Hence the proof of Theorem 7 can be applied without change. For $r=s=0$ we obtain the only interesting conclusion:

TheOREM 10. (The Corona Theorem). If $f_{1}, \cdots, f_{N} \in H^{\infty}$ and (2)' is valid, it follows that $f_{1}, \cdots, f_{N}$ are generators for $H^{\infty}$.

It remains to discuss the proof of Lemma 9. Since the set of bounded functions $\psi$ with $\partial \psi / \partial \bar{z}$ satisfying (8) is a ring, the standard technique for constructing partitions of unity can be applied to derive Lemma 9 from

LEMMA 11. There exists a constant $k$ such that if $0<\epsilon<\frac{1}{2}$ and $f \in H^{\infty}$, $\sup |f| \leqq 1$, one can find $\psi$ with $0 \leqq \psi \leqq 1$ so that $\partial \psi / \partial \bar{z}$ satisfies (8) and 


$$
\psi(z)=0 \text { when }|f(z)|<\epsilon^{k}, \quad \psi(z)=1 \text { when }|f(z)|>\epsilon .
$$

This lemma was proved in a different formulation in [1] when $f$ is a Blaschke product. In fact, the main point in [1] is a construction of certain curves $\Gamma$ surrounding the zeros of a Blaschke product and satisfying conditions which mean precisely that the characteristic function $\psi$ of the exterior of $\Gamma$ has the properties stated in Lemma 11. Since the proof given in [1] is applicable to arbitrary $f \in H^{\infty}$ and we have no significant simplification to contribute, we shall not carry out the proof here.

\section{REFERENCES}

1. L. Carleson, Interpolation by bounded analytic functions and the corona problem, Ann. of Math. (2) 76 (1962), 547-559.

2. L. Hörmander, $L^{2}$ estimates and existence theorems for the $\bar{\partial}$ operator, Acta Math. 113 (1965), 89-152.

3. - An introduction to complex analysis in several variables, D. Van Nostrand, Princeton, N. J., 1966.

4. - $L^{p}$ estimates for (pluri-) subharmonic functions, Math. Scand. 20 (1967), 65-78.

5. J. Kelleher and B. A. Taylor, An application of the corona theorem to some rings of entire functions, Bull. Amer. Math. Soc. 73 (1967), 246-249.

6. B. Malgrange, Sur les systèmes differentiels a coefficients constants, Coll. Int. du Centre National de la Recherche Scientifique, Paris, 1963, pp. 113-122.

Institute for Advanced Study 